\title{
Análisis de las normas internacionales de información financiera y su relación con la responsabilidad social empresarial
}

Rubio-Rodríguez, Gustavo Adolfo; Hernández Aros, Ludivia; Bermeo Pazmiño, Katina Vanessa; Meneses Varón, Nayive Tatiana

Análisis de las normas internacionales de información financiera y su relación con la responsabilidad social empresarial

Revista Economía y Política, núm. 31, 2020

Universidad de Cuenca, Ecuador

Disponible en: http://www.redalyc.org/articulo.oa?id=571162102016

DOI: https://doi.org/10.25097/rep.n31.2020.08

La Universidad de Cuenca en Ecuador, conserva los derechos patrimoniales (copyright) de las obras publicadas, y favorece y permite la reutilización de las mismas bajo la licencia Creative Commons Atribución-NoComercialCompartirlgual 4.0 Internacional (CC BY-NC-SA 4.0), por lo cual se pueden copiar, usar, difundir, transmitir y exponer públicamente, siempre que: a. Se cite la autoría y fuente original de su publicación (revista, editorial, URL y DOI de la obra). b. No se usen para fines comerciales u onerosos. c. Se mencione la existencia y especificaciones de esta licencia de uso.

Esta obra está bajo una Licencia Creative Commons Atribución-NoComercial-Compartirlgual 4.0 Internacional. 
Artículos

\section{Análisis de las normas internacionales de información financiera y su relación con la responsabilidad social empresarial}

Analysis of international financial information rules and their relationship with corporate social responsability

Gustavo Adolfo Rubio-Rodriguez

Corporación Universitaria Minuto de Dios, Colombia

gustavo.rubio-r@uniminuto.edu.co

(iD http://orcid.org/0000-0002-6582-2481

Ludivia Hernández Aros

Universidad Cooperativa de Colombia, Colombia

ludivia.hernandez@campusucc.edu.co

iD http://orcid.org/0000-0002-1571-3439

Katina Vanessa Bermeo Pazmiño

Universidad Católica de Cuenca, Ecuador

kbermeo@ucacue.edu.ec

(iD) http://orcid.org/0000-0002-4438-7855

Nayive Tatiana Meneses Varón

Universidad Cooperativa de Colombia, Colombia

nayive.menesesv@campusucc.edu.co

\section{RESUMEN:}

El objetivo de este manuscrito consiste en analizar la relación que presentan las Normas Internacionales de Información Financiera (NIIF) en relación con las prácticas de Responsabilidad Social Empresarial (RSE) empleadas en el sector empresarial. Este artículo acudió al análisis documental o de contenido, donde se revisaron los antecedentes y ciertas características de la responsabilidad social empresarial, y la correspondencia entre las normas internacionales de información financiera y las memorias de sostenibilidad. Entre los hallazgos más significativos, se logró evidenciar que las empresas presentan información contable y financiera bajo las NIIF, en el que discriminan el tratamiento para cada uno de los hechos económicos de la entidad, pero no detallan políticas de RS, ni cuanto costo incurren por desarrollar sus actividades en materia de RS.

Palabras ClaVe: NIIF, políticas contables, responsabilidad social empresarial.

\section{Abstract:}

The purpose of this manuscript is to analyze the relationship presented by the International Financial Reporting Standards (IFRS) in relation to the Corporate Social Responsibility (CSR) practices used in the business sector. This article went to the documentary or content analysis, where the background and certain characteristics of corporate social responsibility were reviewed, and the correspondence between international financial reporting standards and sustainability reports. Among the most significant findings, it was possible to show that companies present accounting and financial information under IFRS, in which they discriminate the treatment for each one of the economic events of the entity, but they do not detail SR policies, nor how much cost they incur. develop their activities in the field of RS.

KEYWORDS: NIIF, accounting policies, corporate social responsibility.

\section{INTRODUCCIÓN}

Las normas internacionales surgen como necesidad para dirimir conflictos en un mundo convulsionado (Bianchetti, 2017), ante esto, se genera un proceso de homogeneidad en la preparación e interpretación 
de la información financiera en las empresas a nivel mundial. Bajo este contexto, Colombia se encuentra en una transición de normas locales a normas internacionales (Vergara, Londoño, Pérez y Torres, 2015), adquiriendo compromiso y responsabilidad para cumplir de forma adecuada con la sociedad y con las organizaciones que se encargan de ejercer control en el uso de las Normas Internacionales de Información Financiera -NIIF- (Becerra y Miguez, 2019). Del mismo modo, con la aplicación de las NIIF se origina la debida incorporación de la Responsabilidad Social Empresarial (RSE), por medio de un íntegro control en las prácticas que realiza la entidad en cada uno de sus procesos, provocando un impacto social, económico y ambiental (Witkowska, 2016).

La RSE permite demostrar las prácticas que realiza una entidad como estrategia organizacional, basadas en la ética y la aplicación de normas, por lo tanto, tienen un papel fundamental en la sociedad y en el entorno en el cual operan, integrando aspectos económicos, sociales, ambientales y laborales (Jerez, Chango y Cejas, 2017). Asimismo, identificar las políticas contables bajo las NIIF en las empresas que se encargan de manejar la RSE, se puede convertir en un sistema de comunicación entre la empresa y su entorno; es por medio de esta información, que se verifica si realmente se está teniendo o no en cuenta el bienestar a nivel económico, social y ambiental, sobre los hechos reales de la empresa (Perdomo, Villa y Dueñas, 2017a).

El presente manuscrito tiene por objeto analizar el impacto que generan las NIIF y su relación con las prácticas de RSE que llevan a cabo las organizaciones; sus resultados permitirán revelar si las empresas del sector colombiano tienen en cuenta las prácticas de RSE, al presentar la información contable bajo los marcos legales. Según Bohórquez (2015a), la implementación de las NIIF hace que las organizaciones incidan en diversos costos o beneficios incrementando transparencia y calidad en la presentación de su información financiera.

\section{REVISIÓN DE Literatura}

\section{Antecedentes y algunas acepciones de responsabilidad social empresarial}

Según la (GTC) Guía Técnica Colombiana 180, que provee directrices y estrategias para que la empresa presente una gestión socialmente responsable, define la Responsabilidad Social Empresarial como un compromiso voluntario que las organizaciones deberán asumir de acuerdo con sus prácticas; se puede aplicar a cualquier tipo de organización, brindando preceptos para establecer, implementar y mantener la mejora continua en cada uno de los procesos de su gestión organizacional (Cárdenas, Velasco \& Cañizares, 2018). De igual forma, Herrera, Cassiani, Cáceres y Palacios (2016), exponen que la RSE adopta una visión más allá del cumplimiento de los requerimientos legales, siendo fundamental respecto a la aplicación de las NIIF, porque define la transparencia, la eficiencia y la ética en los resultados de los estados financieros.

La RSE permite identificar si una organización verdaderamente aplica una cultura socialmente responsable, conformada por valores éticos que puedan ser implementados en cada proceso del buen gobierno corporativo, como lo son: brindar una información con base en hechos económicos reales, inversiones responsables, relación equilibrada y transparente con los grupos de interés, y gestión eficientemente responsable de los riesgos sociales, económicos y medioambientales (Alija, 2017).

En la actualidad, las empresas colombianas incorporan la responsabilidad social de una manera socio ambiental, e involucran estrategias de sostenibilidad dentro de sus prácticas en un mediano y largo plazo (Toca, 2017). Según, Bertolotti (2016), la RS es un recurso indispensable para el desarrollo sostenible en la medida en que se vaya incorporando, ya que busca alcanzar objetivos más allá de lo económico o del beneficio organizacional que se pueda proporcionar, enfocándose hacia la necesidad de un verdadero cambio e impacto social y ambiental, garantizando un desarrollo sustentable e integral con la sociedad. 
La gestión de la RSE es un proceso que se ha involucrado tanto en las obligaciones como en los compromisos sociales y éticos de una organización (Herrera, Larran, Lechuga y Martínez, 2015). La importancia de los compromisos sociales que se deben mantener en las prácticas de RSE, radica en que la información y las actividades desarrolladas por la entidad, se den a conocer a los grupos de interés (stakeholders), considerándose como una política estratégica a nivel social, ya que se debe mantener una relación de satisfacción con la información suministrada a los usuarios interesados, alcanzando un alto grado de transparencia y reputación con los resultados obtenidos en la empresa (Solano, Plaza y Flores, 2015).

Cabe resaltar que, en Colombia, las empresas han venido avanzando en la incorporación de políticas para el buen gobierno corporativo y la implementación de estrategias en tema de RSE, que puede brindar beneficios internos y externos (Salamanca, 2016). En este contexto, Rodrigo \& Arenas (2014) argumentan que los informes de RSE se han convertido en una revelación más para las organizaciones, ya que influyen en la toma de decisiones para futuras proyecciones. Sin embargo, Macías (2018), expone que hoy en día muchas empresas relacionan la RSE con medio ambiente, pero más que conservar el medio ambiente, las empresas deben tener en cuenta la forma en la que impactan de manera económica, social y ambiental, su entorno.

Las estrategias en materia de RSE que actualmente emplean las empresas colombianas, se ven orientadas al crecimiento patrimonial y cumplimiento de los objetivos económicos y financieros (Marquina y Reficco, 2015); no obstante, Ramírez (2017), refiere que en los últimos años se ha evidenciado que las organizaciones colombianas deben tener en cuenta los intereses tanto de la entidad, inversionistas, proveedores, trabajadores, de la comunidad en general, que afectan ya sea de manera directa e indirecta en el desarrollo social. Es indispensable que las empresas logren las metas propuestas para que las prácticas sean sostenibles con el tiempo y así crear un compromiso por parte del gobierno corporativo, para que las políticas estratégicas estén orientadas en un propósito común (Contreras, Avella y Gómez, 2015).

\section{Normas internacionales de información financiera y su correspondencia con las memorias de sostenibilidad}

Las NIIF son un conjunto de normas contables emitidas por el Consejo de Normas Internacionales de Contabilidad (IASB), con la finalidad de brindar transparencia, compromiso y eficacia en los mercados financieros (IFRS, 2017). Según Garza, Cortez, Méndez y Rodríguez (2017), el marco conceptual del IASB pretende unificar normas contables para el direccionamiento y fundamento de las normas emitidas por organismos internacionales, mostrando control en la emisión de la información financiera. Con la aplicación de las normas contables a nivel mundial, las empresas hablarán un solo idioma por medio de la información contable presentada de forma relevante y de alta calidad (Legaz, Montoya y Rodríguez, 2015) y especialmente bajo los mismos estándares de medición, valoración y revelación.

Asimismo, se puede establecer que la demanda financiera de las empresas actualmente está regida por los efectos de la implementación de las NIIF (Martínez, 2017), por tanto, las normas internacionales inciden en la sostenibilidad corporativa, proporcionando una buena gestión en la medida en la que se reestructuran las políticas y estrategias internas y externas de la organización, con la finalidad de dar a conocer el efecto que producen a la sociedad y a la economía, y así demostrar su transparencia en la gestión de sus actividades (Polanco, Ramírez y Orozco, 2016). Para lograr un buen desarrollo económico y control en las organizaciones, deben estar a cargo de profesionales íntegros, con conocimientos adecuados para el desempeño de sus funciones, ejerciendo su actividad bajo la normatividad y ética que los rige (Minatti, Borba, Aguilar y Rodrigues, 2014).

Por medio de las memorias de sostenibilidad, la organización puede compartir información o rendir cuentas a grupos de interés internos y externos en relación con el desarrollo sostenible. De acuerdo a la Guía para la elaboración de memorias de sostenibilidad (GRI, 2015), algunas empresas presentan este informe 
como un adicional, más no, como un informe obligatorio (Sepúlveda, Valero y Bonilla, 2018a); las memorias de sostenibilidad sirven como apoyo a las empresas al momento de establecer sus objetivos, evaluar su rendimiento y plantear nuevas políticas estratégicas para llegar a ser sostenible (Mantilla, Cabeza y Vargas, 2015).

Algunas empresas colombianas del sector privado, han elaborado voluntariamente memorias o informes de sostenibilidad, en los que se pueden observar sus proyecciones de acuerdo a su objeto social sin perder de vista los intereses de los grupos de interés con los que se relacionan (Rodríguez, Frías y García, 2014). Las memorias de sostenibilidad, proponen equilibrar los objetivos de protección ambiental, crecimiento económico y bienestar de la sociedad en general, sin afectar el bienestar del futuro, y alcanzar las metas de crecimiento y productividad soportados por la información financiera de la empresa (Déniz y Verona, 2015).

Según Villanueva, Zorio y García (2015), las empresas son las que se benefician por la explotación de los recursos naturales para la producción de sus bienes y servicios, así como los residuos generados durante los procesos productivos, sin tener en cuenta el deterioro que puedan estar ocasionando al medio ambiente, lo que puede afectar la sostenibilidad de la sociedad y de la misma organización. El IASB, ha emitido normas internacionales para el debido registro y tratamiento contable relacionado con el medio ambiente y los recursos naturales (Lasso, Vargas y Ruano, 2018); esta interpretación, se traduce a un reconocimiento en los estados financieros derivados de las obligaciones o aprovechamiento de los recursos naturales (Alonso, Marimon y Llach, 2015).

Las Normas Internacionales de Información Financiera, según Boz, Menéndez, Orgaz y Prior (2015), tienen como finalidad establecer técnicas para el reconocimiento y presentación de criterios contables, en las que se involucra la organización, actuando de manera transparente al presentar informes financieros. De igual manera, con la aplicación de las NIIF las organizaciones deben establecer políticas contables para el debido tratamiento de la información (Patiño y Sepúlveda, 2017). Demostrando una revelación y medición, de acuerdo al tratamiento que la norma le permita respecto al desarrollo de su actividad económica (Hernández, 2015), las empresas deben responsabilizarse por adoptar normas internacionales relacionadas con el desarrollo sostenible, según sea el caso, soportando el ejecutar de las operaciones con la información del impacto que se produce al medio ambiente, la economía y la sociedad, sin dejar de informar la recuperación o la prevención en pro del mismo (Cuadrado, García y Martínez, 2015).

\section{Diseño Metodológico}

El presente manuscrito utilizó un nivel de análisis descriptivo y explicativo, pues, de acuerdo a Hernández, Fernández y Baptista (2014), se refieren a que "los estudios descriptivos se centran en recolectar datos que muestran un evento, una comunidad, un fenómeno, hecho, contexto o situación" (p. 115). Para el efecto, se acudió por una parte a la revisión documental, haciendo énfasis en la conceptualización y contextualización de las dos variables objeto de estudio, es decir, las Normas Internacionales de Información Financiera (NIIF), y Responsabilidad Social Empresarial (RSE).

Para ello, se tuvo en cuenta las siguientes fases: Fase 1) Mediante una exploración bibliográfica se realizó la búsqueda de información necesaria para llevar a cabo el desarrollo del tema descrito. Fase 2) Una vez realizada la búsqueda en bases de datos, se analizó la relación entre las NIIF y la RSE con base en normas e investigaciones de artículos extraídos de revistas indexadas. Fase 3) Se presentó en una tabla, el impacto que generan las NIIF en las políticas de responsabilidad social en una organización.

Las limitaciones del análisis en la temática estudiada, radican en la carencia de estudios investigativos que antecedan el tema objeto de estudio. Luego de examinar considerablemente diferentes bases de datos, no se encontró con suficiencia, un trabajo investigativo que precisará la relación que presentan las Normas Internacionales de Información Financiera (NIIF) en relación con las prácticas de Responsabilidad Social Empresarial (RSE) empleadas en el sector empresarial. Sin embargo, la información contenida en este 
manuscrito se infiere cuidadosamente de la recolección de datos de fuentes confiables, los cuales fueron analizados por separado, pero logrando alcanzar el cumplimiento del objetivo propuesto.

\section{Discusión de Resultados}

Para responder al objeto por el cual se generó este trabajo, primero se presentará la relación existente entre las NIIF y la RSE; seguidamente, se mostrará la identificación de las NIIF y las principales políticas contables en las empresas que practican RSE.

\section{Relación entre las NIIF y la RSE}

Para evidenciar la relación que se existe entre las NIIF y la RSE, la Tabla 1 enseña esta relación, según algunas pesquisas desarrolladas por investigadores, y la normatividad que la regula.

TABLA 1.

Relación entre las NIIF y la RSE

\begin{tabular}{ll}
\hline Estudios y/o Normas & Relación entre las NIF y la RSE \\
\hline Ley 1314 (2009) & $\begin{array}{l}\text { Los requerimientos para la preparación de la información } \\
\text { contable, exige a las organizaciones atender sus actividades } \\
\text { en función de la sociedad. }\end{array}$ \\
\hline $\begin{array}{l}\text { Puerta, Vergara y Huertas } \\
\text { (2018) }\end{array}$ & $\begin{array}{l}\text { Al administrar de manera adecuada las normas } \\
\text { internacionales, las empresas son responsables económica, } \\
\text { ambiental y socialmente. }\end{array}$ \\
\hline Mancilla y Saavedra (2015) & $\begin{array}{l}\text { En la medida en que se unifiquen criterios, técnicas } \\
\text { contables y prácticas de Rs, la uniformidad de la } \\
\text { información contable es viable. }\end{array}$ \\
\hline Rendón (2018) & $\begin{array}{l}\text { La implementación de las NIIF no solo aplica al ejercicio } \\
\text { contable, sino es esencial, en los procesos de adaptación y } \\
\text { capacitación para el personal. }\end{array}$ \\
\hline Amezquita, López y & $\begin{array}{l}\text { La elaboración de políticas que consientan la } \\
\text { implementación de las NIIF permite la regulación contable y } \\
\text { Social que esto implica. }\end{array}$ \\
\hline Solano (2017) & $\begin{array}{l}\text { la RSE se percibe como un impacto económico, social y } \\
\text { ambiental adecuado para proporcionar información } \\
\text { financiera, }\end{array}$ \\
\hline Martínez, Prado y Fernández & $\begin{array}{l}\text { La RSE es una nueva estrategia organizacional; esta debe } \\
\text { velar, entre otros, por reflejar transparentemente } \\
\text { información contable y financiera. }\end{array}$ \\
\hline Castilla y Gallardo (2014) & $\begin{array}{l}\text { La integración de la información financiera en la sociedad, } \\
\text { involucra una responsabilidad social y transparencia }\end{array}$ \\
& profesional. \\
\hline Bonito y Pais (2018) & $\begin{array}{l}\text { Es imperioso que se establezcan normas de contabilidad e } \\
\text { información financiera, con las cuales se enseñe } \\
\text { responsablemente la realidad económica de la empresa. }\end{array}$ \\
\hline García y Serpa (2015) & $\begin{array}{l}\text { Las NIIF deben contribuir a que las empresas incorporen } \\
\text { dentro de sus políticas y objetivos organizacionales la RS, a } \\
\text { través de los sistemas de información contable. }\end{array}$ \\
\hline
\end{tabular}

Elaboración propia

La convergencia contable internacional de la información financiera, tiene como criterio fundamental, la implementación de normas nacionales a normas internacionales; para Colombia a partir del año 2009, entró la ley 1314 en la que se establecen principios y requerimientos para la preparación de la información contable conformada por el reconocimiento, medición y revelación de los hechos económicos (Ley 1314, 2009). De igual manera, la reglamentación es la uniformidad entre los sistemas y la presentación de la información 
financiera, proporcionando entendimiento a las necesidades de los usuarios interesados en esta información, lo que sumerge a la empresa en una Responsabilidad Social, ya que desarrolla sus actividades en la sociedad y para el bienestar de la misma (Bohórquez, 2015b).

Además de lograr una unificación de las NIIF, se encuentra la aplicación de la RSE, considerando que estas dos se relacionan entre sí, teniendo en cuenta que las empresas deben ser responsables económica, ambiental y socialmente, al aplicar de manera correcta las normas internacionales para la preparación de la información financiera (Puerta, Vergara y Huertas, 2018a). Así lo soportan Mancilla y Saavedra (2015), cuando afirman que la uniformidad de la información contable es viable en la medida en que se unifiquen criterios, técnicas contables y prácticas de RS, permitiendo la comparabilidad y la competitividad de la organización a nivel internacional; también, se considera que la unificación de las NIIF permite que la empresa acceda a mercados financieros representativos para el beneficio de ésta.

Como se mencionó anteriormente, con la ley 1314, se establecieron principios y normas de contabilidad y de aseguramiento en Colombia, cuyo objetivo principal es que las empresas mejoren su productividad, competencia y relación con los usuarios internos y externos, ya sea a nivel nacional o internacional (Rendón, 2018); no obstante, de acuerdo a lo acotado por Amezquita, López y Villanueva (2015), la implementación de las NIIF no solo es para el ejercicio contable, por el contrario, es fundamental, ya que brindan a las empresas un proceso de adaptación y capacitación para el personal, y con ello un desarrollo de políticas que permitan que las NIIF sean aplicadas de manera correcta, sin perder de vista la regulación contable y social que implica.

Según las NIIF, la contabilidad se puede definir como una técnica que se utiliza para el proceso de registro y reconocimiento de los hechos económicos y operaciones que afectan de manera económica a la organización, y que genera sistemática y estructuradamente la información financiera, reflejando aspectos internos, externos, transacciones y hechos reales, donde la RSE se percibe como un impacto económico, social y ambiental adecuado para proporcionar información financiera, presentada a través de estados financieros ejecutados con las normas vigentes para la toma de decisiones de usuarios interesados en dicha información (Solano, 2017).

Actualmente, las organizaciones colombianas se ven expuestas a una demanda por parte de la sociedad, respecto a los impactos que presentan a nivel económico y social a través de la RSE (Perdomo, Villa y Dueñas, (2017b). Según Martínez, Prado y Fernández (2013), la RSE es una nueva estrategia organizacional que va más allá de un requerimiento legal, y se aplica de manera voluntaria, lo que traduce a que las empresas ya no sean competitivas por el servicio o producto que brindan a la sociedad, sino, por la mejora en sus políticas y herramientas estratégicas, reflejando en su información contable y financiera, el tratamiento que la entidad aplica respecto al impacto que produce al desarrollo sostenible, ya sea de manera negativa o positiva, dependiendo de la actividad que desarrolle para el bienestar de la misma.

Frente a este panorama, la integración de la información financiera en la sociedad, en aspectos globales e internacionales, involucra una Responsabilidad Social Empresarial y transparencia profesional (Castilla y Gallardo, 2014), en la medida en que se reconoce que, además de una Responsabilidad Social con la misma empresa, esta genera consecuencias en la calidad de vida de la comunidad y sus empleados (Vaz, Ruiz y Fernández, 2018). Bonito y Pais (2018), argumentan que en los estándares internacionales emitidos por la IASB, es necesario que los profesionales encargados de aplicar y desarrollar determinada reglamentación, establezcan normas de contabilidad e información financiera y de aseguramiento de la información, lo que permite que el sistema de información sea de alta calidad con el propósito de que, al momento de ejecutarse en las organizaciones, refleje la realidad económica de la empresa.

La aplicación de políticas de RSE se ha convertido en una estrategia para fortalecer la gestión de las empresas desde el ámbito social y económico, buscando beneficios significativos para la organización (Sanchis y Rodríguez, 2018); por lo tanto, el contador público mediante su responsabilidad y ética profesional se convierte en garante de transparencia y confianza, y enfoca su visión en el manejo adecuado de los recursos de una entidad de acuerdo al entorno económico y social que desarrolle la empresa. Según Ruiz (2018), 
las NIIF no le exigen al profesional contable una preparación y capacitación en el nuevo marco normativo en el que se ven involucradas todas las empresas colombianas, pero de acuerdo a la normatividad, todas las empresas que lleven contabilidad deben estar expuestas a este cambio, lo que obliga de manera indirecta al contador a capacitarse y actualizarse en dicha regulación, para generar independencia y objetividad al ejercer esta disciplina.

Las NIIF deben contribuir a que las empresas constantemente incorporen dentro de sus políticas y objetivos organizacionales la RS a través de los sistemas de información contable, que permitan realizar un control y seguimiento al manejo de los recursos y el impacto económico, social y ambiental de una manera interna y externa (García y Serpa, 2015). La información contable que permita brindar aseguramiento sobre la divulgación de las prácticas de RSE, es una información adecuada, pertinente, útil y transparente con base en las características de una empresa socialmente responsable.

\section{Identificación de las NIIF y principales políticas contables en las empresas que practican RSE}

Las empresas han incorporado en la presentación de su información financiera documentos que permiten identificar el reconocimiento, mismos que están facilitando la aplicación de nuevas políticas en materia de RSE; uno de ellos es el Balance Social, el cual es un informe que permite determinar el impacto social y ambiental de las actividades que está desarrollando la empresa en términos cuantitativos y cualitativos en su gestión por determinado periodo de tiempo y de acuerdo a los objetivos principalmente establecidos (Sepúlveda, Valero y Bonilla, 2018). Cuando la organización implementa las NIIF en la presentación de los estados financieros, logran accesibilidad al sector internacional, obteniendo como resultado beneficios potenciales, eficiencia en sus procesos y aumento patrimonial que favorece a la organización (Puerta, Vergara y Huertas, 2018b).

Del mismo modo, en la presentación de la información financiera se deben definir políticas contables que permitan ofrecer lineamientos o procedimientos para tener en cuenta al momento de presentarla (Escobar, 2016); por consiguiente, deben reflejar las proyecciones o actividades sociales y ambientales, con los cuales la organización busca disminuir el impacto que ha generado de manera positiva en el desarrollo de su objeto social (Contreras, Pedraza y Martínez, 2017). Se puede evidenciar que las NIIF no especifican como las empresas deben manejar procesos relacionados con el impacto socio-ambiental o las actividades que realicen en pro de la misma, pero si detalla, el manejo que le debe dar a los rubros contables expuestos en los estados financieros de manera general y de esta forma medir el nivel de responsabilidad social que se practica (Alarcón y Álvarez, 2018).

La información financiera y contable que rinde cuentas de la responsabilidad social, debe permitir que la organización presente estados financieros con base en hechos reales desde un ámbito social, económico y ambiental (Alarcón, Valenzuela, Gutiérrez y Gil, 2015), de tal manera, informará a los stakeholders para que tomen decisiones tanto de inversión, consumo o aumento patrimonial para las partes. De ahí, la importancia de que el gobierno corporativo realice estrategias y estructure políticas contables, para el debido reconocimiento de las prácticas que realiza la entidad en pro de la RSE, contribuyendo al beneficio de la entidad para que sea sostenible y aporte en el bienestar de la población en general (Fernández, 2018).

Las NIIF permiten que las entidades manejen una información contable y financiera entendible para otros usuarios, desde un mercado nacional e internacional; por consiguiente, dicha información debe reflejar las prácticas de RSE para ser más competitivo e innovador, y esto se obtiene con una buena gestión y organización de las actividades que desempeña (Ardila, García, y Ardila, 2016).

Las NIIF han generado un gran impacto en las empresas no solo en lo contable, sino también, en todas las actividades que desarrolla la entidad por medio de la divulgación de las prácticas de RSE (Volpentesta, 
2017); con la implementación de las NIIF, las políticas y estrategias que estructure la empresa para ejecutar las prácticas de RS, se puede evidenciar en algunas de las normas y técnicas que permiten que las empresas presenten sus estados financieros de manera transparente y real, de acuerdo a sus prácticas en el entorno donde desarrollan sus actividades y reflejando cumplimiento a la normatividad (De la cuesta, Pardo y Paredes, 2015).

La NIIF 7 es una norma muy importante para la organización, ya que permite identificar los ingresos y gastos en los que incurre la empresa para dar consecución a los requerimientos leales, y hechos económicos que afectan de alguna forma la RS (NIIF 7); la NIIF 6 permite clasificar los hechos económicos en los que incurren las empresas en el reconocimiento de los activos para exploración y evaluación de recursos minerales, para que ayude a los Stakeholders a entender el manejo y tratamiento para de dichos activos (NIIF 6); por otra parte, la NIIF 19 "tratamiento para los beneficios a empleados", establece políticas que le faciliten a la empresa crear estrategias para motivar al personal a producir responsablemente impartiendo una gestión de alta calidad en el control y manejo del recurso humano (NIIF 19).

Por lo tanto, las NIIF han generado un impacto en la divulgación y aplicación de la RSE; es por ello, la necesidad de que las empresas estructuren y creen estrategias para el diseño de políticas contables con base en la información que presenten las prácticas de RSE en las cuales incurren las organizaciones (Quintero, 2017).

A continuación, a manera de ejemplo, la Tabla 2 presenta el impacto que generan las NIIF en las políticas de responsabilidad social en una organización.

TABLA 2.

Impacto de las NIIF en Políticas de Responsabilidad Social Empresarial

\begin{tabular}{|c|c|c|}
\hline Politicas de Responsabilidad Social Empres arial & Afectacion & NIIF \\
\hline 1) Gestión responsable del consumo de agua y energia & Estado de resultados- Ingresos & NIIF 7. Instrumentos financieros: información a revelar NIIF 9 . Instrumentos financieros \\
\hline 2) Combatir el hambre & Estado de resultados-Deducción por que genera gastos : & NIIIF 7. Instrumentos financieros: informacion a revelar NIIF 9 . Instrumentos financieros \\
\hline 3) Apoyo a los empleados & A fectan la NIIF de empleadcs & NIIF 7 Instrumentos financieross: información a revelar vilc 19 . Eeneficios a empleados \\
\hline 4) Apoyo a los proveedores & Dismininución de costos & NIIF 7. Instrumentos financieros: información a revelar NIIF 9. Instrumentos financierros NIIF 11. Acuerdos conjuntos \\
\hline 5) Creación de fundaciones & Aumento de activos y estado de situación económica & NIIF 7. Instrumentos financieros: información a revelar NIIF 9 . Instrumentos financiercs NIIF 12. Información a revelar sobre participaciones en otras entidades \\
\hline 6) Programas de alfabetización & Estado de resultados & WIIF 7. Instrumentos financieros: informacion a revelar NIIF 9 . Instrumentos financieros \\
\hline 7) Apoyo para mejorar las condiciones esucativas & Inversiones y gastos & 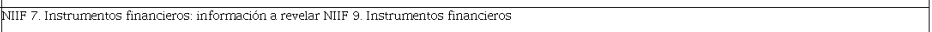 \\
\hline 8) Mejoramiento de la infraestructura social & Inversión & WIIF 7. Instrumentos financieros: información a revelar NIIF ९. Instrumentos financieros \\
\hline 9) Sensibilización de cuicacio al medio ambiente & Ingresess & 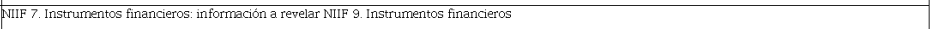 \\
\hline 10) Gestión integra de los residuos & Ingresos & NIIF 7. Instrumentos financieros: información a revelar NIIF 9. Instrumentos financieros NIIF 13. Mediciön del valor razonable \\
\hline 11) Generarar valor a los grupos de interés & Clientes-Acciones & VIIF 2 Fagos basados en acciones SiliF 7. Instrumentos financieros: información a revelar \\
\hline
\end{tabular}

Elaboración propia, adaptada de Calderón, Mora y Sierra, (s.f.)

\section{Conclusiones}

Las NIIF inciden en las prácticas de RSE en la medida en la que las empresas incorporen políticas que contribuyan a la buena gestión de la RSE, a través de un eficiente sistema de información contable; las prácticas, políticas contables y de RSE deben reflejar las actividades con las que la empresa pretende disminuir el impacto negativo que pueda estar generando en el desarrollo de su objeto social. Sin embargo, las NIIF no relacionan de manera directa como se debe reconocer estos procesos o prácticas, pero están inmersos en el manejo que se le debe dar a los gastos en los que incurren las organizaciones que se reflejarán en los estados 
financieros y el resultado de la actividad con el que se medirá la RSE ejecutada, por consiguiente, no se puede llegar a obtener de manera detallada el proceso de cumplimiento de RSE.

La RSE es un factor muy importante en una organización, esto en razón de la globalización y los cambios ambientales en los que cada vez se ven más involucradas las actividades que desarrollan las entidades para dar cumplimento a su objeto social; hay que resaltar que, aunque la RSE genera gastos para la empresa, estos deben reconocerse como una inversión, ya que las estrategias que se dispongan para llevar a cabo una buena gestión respecto a la RSE, de forma directa o indirecta, proporcionan una mayor rentabilidad para la empresa en la medida en que su participación en la sociedad se haga denotar de manera positiva, aportando un valor agregado en el desarrollo de sus actividades.

Las prácticas de RSE deben estar reflejadas en los estados financieros de las empresas con base en las políticas tanto contables como social, económica y ambientalmente; se puede evidenciar que las empresas que están comprometidas con la RS e incluyen políticas para su debido tratamiento, alineadas con los proyectos estratégicos y la cultura interna de la organización, tienen como resultado un cambio significativo en el mejoramiento de su desempeño tanto contable como administrativo. De igual forma, los beneficios de la RSE deben reflejarse en la información presentada ante la sociedad, para que sea participe de los beneficios, avances y actuaciones en la aplicación de políticas para el control y registro de los hechos económicos que se detallen fielmente en los estados financieros.

Finalmente, la reflexión de este trabajo invita no solo a investigadores sino a organizaciones encargadas de emitir políticas y técnicas contables, a que se amplíe este tema abordado. Respecto al análisis realizado en diferentes informes financieros de empresas anteriormente mencionadas, se logra evidenciar que no presentan de manera detallada sus políticas ni valores en los que las entidades incurren para cumplir con la RSE, por lo tanto, se sugiere que organismos reguladores de la información contable y financiera expidan normas para que las empresas incorporen políticas para el manejo de la RSE e informen en sus estados financieros, de manera detallada, cuanto les cuesta o cuanto invierten en materia de RSE tanto a nivel nacional como internacional.

\section{Referencias Bibliográficas}

Alarcón, C., Valenzuela, L., Gutiérrez, A., \& Gil, J. (2015). Sensibilidad ética empresarial. Innovar, 25(58). Recuperado de https://bbibliograficas.ucc.edu.co:2149/docview/1732083443?accountid=44394\&rfr_id=inf o\%3Axri\%2Fsid\%3Aprimo

Alarcón, M., \& Álvarez, J. (2018). La dimensión sectorial del cooperativismo colombiano a través de un análisis de redes. CIRIEC, 92. Recuperado de https://bbibliograficas.ucc.edu.co:2149/docview/2059070132/fulltextPD F/DEB95BD5EEC147E7PQ/1 ?accountid=44394

Alija, T. (2017, 12 agosto). Enfoques y perspectivas económicas y sociales de la responsabilidad corporativa. Sistema de Información Científica Redalyc, 33(58). Recuperado de https://www.redalyc.org/jatsRepo/2250/2250569 47008/index.html

Alonso, M., Marimon, F., \& Llach, J. (2015). Difusión de las memorias de sostenibilidad en Latinoamérica análisis territorial y sectorial. Estudios Gerenciales: Journal of Management and Economics for Iberoamerica, 31(135). Recuperado de https://dialnet.unirioja.es/servlet/articulo?codigo $=5058763$

Amezquita, D., López, L., \& Villanueva, M. (2015). Estrategia para la adopción de las niif en la PYMES de Tunja. Saber, ciencia y libertad, 10(2). Recuperado de https://dialnet.unirioja.es/servlet/articulo?codigo $=5329122$

Ardila, F., García, D., \& Ardila, J. (2016). Rediseño e implementación de las NIIF en las PYME. Análisis Financiero. Recuperado de https://dialnet.unirioja.es/servlet/articulo?codigo $=5782366$

Becerra, J. \& Miguez, L. (2019). Impactos en el proceso de convergencia de las Normas Internacionales de Información Financiera para Pymes en una empresa del sector de transportes: estudio de caso. Gestión Joven, (19), 1-21. 
Bertolotti, M. (2016). Sostenibilidad y pesca responsable: Las dimensiones social y económica, principios, objetivos e indicadores/Sustainability and responsible fisheries: Social and economic dimensions, principles, aims and indicators. Frente Maritimo, 24, 11-29. Recuperado de https://bbibliograficas.ucc.edu.co:2149/docview/1795 669211 ? accountid $=44394$

Bianchetti, L. (2017). El proceso de Bolonia y la globalización de la educación superior. CLACSO: Repositorio Digital, 91 pp. _. RIED. Revista Iberoamericana de Educación a Distancia, 20(2), 339-344.

Bohórquez, M. (2015). Implementación de norma internacional de inventarios en Colombia. Innovar, 27(52). Recuperado de https://dialnet.unirioja.es/servlet/articulo?codigo $=5129207$

Bonito, A., \& Pais, C. (2018). Los determinantes macroeconómicos de la adopción de las NIIF para las PYMES. Revista de contabilidad, 21(2). Recuperado de https://www.sciencedirect.com/science/article/pii/S11384891183000 13

Boz, G., Menéndez, C., Orgaz, N., \& Prior, D. (2015). ¿Influyen las Normas Internacionales de Información Financiera en el riesgo de las acciones? Contaduría y Administración, 60(0). Recuperado de https://www.sciencedirect.co $\mathrm{m} /$ science/article/pii/S0186104215000145

Cárdenas, M., Velasco, B., Burgos, \& Cañizares, J. (2018). Coopetencia, modelo de Gestión Empresarial para Mipymes de Ocaña. Respuestas, 23(1), 59-65.

Castilla F., \& Gallardo D. (2014). La revelación social en sociedades cooperativas1: una visión comparativa de las herramientas más utilizadas en la actualidad. Revesco: Revista De Estudios Cooperativos, (114), 7-34. Recuperado de https://bbibliograficas.ucc.edu.co:2149/docview/1524709455?accountid=44394

Contreras, O. E., Avella, A. C. P., \& Gómez, C. H. (2015). La inclusión social de la fuerza laboral en colombia. ¿En contravía de lo sostenible? */Is social inclusion of workforce in colombia in counter of what sustainability means?/A inclusão social da força de trabalho na colômbia É isto contrario do sustentável? Apuntes Del CENES, 34(60), 131-152. Retrieved from https://bbibliograficas.ucc.edu.co:2149/docview/1715656117?accountid=4 4394

Contreras, O., Pedraza, A., \& Martínez, M. (2017). La inversión impacto como medio de impulso al desarrollo sostenible: una aproximación multicaso a nivel de empresa en Colombia. Estudios Gerenciales, 33(142). Recuperado de https://www.sciencedirect.com/science/article/pii/S0123592317300074

Cuadrado, B., García, R. \& Martínez, J. (2015). Efecto de la composición del consejo de administración en las prácticas de responsabilidad social corporativa. Revista De Contabilidad, 18(1), 20-31. Recuperado de https://bbibliogr aficas.ucc.edu.co:2149/docview $/ 1670115972$ ?accountid $=44394$

De la cuesta, M., Pardo, E., \& Paredes, J. (2015). Identificación de indicadores relevantes del desempeño rse mediante la utilización de técnicas multicriterio. Innovar, 25(55). Recuperado de https://bbibliograficas.ucc.edu.co:2149 /pqrl/docview/1676945924/fulltextPDF/58642A2E048E4204PQ/82?accountid=44394

Déniz, J., \& Verona, M. (2015). Deconstruyendo el resultado contable convencional para diseñar un resultado contable ambiental. Contaduría y administración, 60(3). Recuperado de https://dialnet.unirioja.es/servlet/articulo?cod igo $=5482240$

Escobar, G. (2016). Crecimiento Económico como determinante de los flujos de efectivo para las empresas del sector industrial de Caldas - Colombia, durante el periodo 1995 - 2013. Ánfora: Revista Científica de la Universidad Autónoma de Manizales, 23(41). Recuperado de https://dialnet.unirioja.es/servlet/articulo?codigo= 5755366

Hernández, R., Fernández, C., y Baptista, M. (2014). Metodología de la Investigación. México: McGraw Hill.

Fernández, T. (2018). Indicadores para la comunicación de la responsabilidad social corporativa de las radiotelevisiones públicas europeas. Prisma social, 22(0). Recuperado de https://bbibliograficas.ucc.edu.co:2149/docview/2190 928657/fulltextPDF/1D9A31FF29146ACPQ/22?accountid=44394

García, M., \& Serpa, I. (2015). Metodología para la conversión de la información contable y financiera hacia la norma NIIF para PYMES. Saber, ciencia y libertad, 10(2). Recuperado de https://dialnet.unirioja.es/servlet/articulo? codigo $=5329132$ 
Garza, H., Cortez, K., Méndez, A., \& Rodríguez, M. (2017). Efecto en la calidad de la información ante cambios en la normatividad contable: caso aplicado al sector real mexicano. Contaduría y Administración. Recuperado de ht tps://www.sciencedirect.com/journal/contaduria-y-administracion

GRI, Global Reporting Initiative (2015). Guía para la elaboración de Memorias de Sostenibilidad. Recuperado de ht tps://www.globalreporting.org/information/about-gri/governance-bodies/Pages/default.aspx

Guía Técnica Colombia 180 (2008). Responsabilidad Social Empresarial. Recuperado de https://tienda.icontec.org/ wp-content/uploads/pdfs/GTC180.pdf

Hernández, D. (2015). Fundamentos teóricos para la construcción de un modelo de contabilidad social. Criterio libre, 26. Recuperado de https://bbibliograficas.ucc.edu.co:2149/docview/1927850861/fulltextPDF/D880C66EC $8 \mathrm{~B} 24432 \mathrm{PQ} / 1$ ? accountid $=44394$

Herrera, F., Cassiani, D., Cáceres, A., \& Palacios, A. (2016). Gobierno corporativo y tendencias actuales en Colombia. Liderazgo Estratégico, 6(1), 65-72. Recuperado a partir de http://revistas.unisimon.edu.co/index.php/liderazg o/article/view/3251

Herrera, J., Larran, M., Lechuga, M., \& Martínez, D. (2015). Responsabilidad social en las pymes: análisis exploratorio de factores explicativos. Revista de contabilidad, 19(1). Recuperado de http://www.elsevier.es/es-revista-revista -contabilidad-spanish-accounting-review-368-pdf-S1138489114000466

IFRS (2015). Internacional Financial Reporting Standards. Proyecto de Norma Marco Conceptual para la Información Financiera. Recuperado de http://www.incp.org.co/Site/2015/info/archivos/proyecto-norma-m arco-conceptual.pdf

Jeréz, M., Chango, M., \& Cejas, M. (2017). La responsabilidad social empresarial: Alcances y Perspectivas en el marco de la Competitividad. http://repositorio.pucesa.edu.ec/handle/123456789/2222

Lasso, G., Vargas, C., \& Ruano, C. (2018). Efecto patrimonial del proceso de convergencia contable en las Pymes colombianas. Estudios Gerenciales, 34(146). Recuperado de https://bbibliograficas.ucc.edu.co:2149/docview/ $2058607951 /$ fulltextPDF/A8E798A0B8544E6CPQ/1 ?accountid $=44394$

Legaz, J., Montoya, J., \& Rodríguez, L. (2015). Efectos de la reforma contable en el patrimonio neto consolidado a 1 de enero de 2008 de los grupos españoles ₹ que no aplican normativa NIIF. Revista de Contabilidad, 18(2). Recuperado de https://bbibliograficas.ucc.edu.co:2152/search/advanced?docId=10.1016/j.rcsar.2015.01.007

Macías, W. (2018). Hacia una resignifi cación del sentido de la responsabilidad social desde la autonomía y solidaridad de las organizaciones campesinas: El caso del comité de integración del macizo colombiano. Criterio Libre, 16(28), 107-131. Retrieved from https://bbibliograficas.ucc.edu.co:2149/docview/2125263511 ?accountid=4 4394

Mantilla, E., Cabeza, M., \& Vargas, J. (2015). La realidad del desarrollo y la contabilidad ambiental. Saber, Ciencia y Libertad, 10(2). Recuperado de https://dialnet.unirioja.es/servlet/articulo?codigo $=5329131$

Mancilla, M., \& Saavedra, M. (2015). El gobierno corporativo y el comité de auditoría en el marco de la responsabilidad social empresarial. Contaduría y administración, 60(2). Recuperado de https://www.sciencedirect.com/scienc e/article/pii/S0186104215300115

Marquina, P., \& Reficco, E. (2015). Impacto de la responsabilidad social empresarial en el comportamiento de compra y disposición a pagar de consumidores bogotanos. Estudios Gerenciales, 31(137). Recuperado de https://www .sciencedirect.com/science/article/pii/S0123592315000558

Martínez F. (2017). Posicionamiento del patrimonio en cooperativas de intermediación financiera frente a las NIIF sobre instrumentos financieros. Clio America, 11(21). Recuperado de: http://bbibliograficas.ucc.edu.co:2076/ $10.21676 / 23897848.2075$

Minatti, F., Borba, J., Aguilar, C., \& Rodrigues, E. (2014). Importancia de las contingencias ambientales una investigación en las empresas brasileñas. Contabilidad y Negocios: Revista del Departamento Académico de Ciencias Administrativas, 9(18). Recuperado de https://dialnet.unirioja.es/servlet/articulo?codigo=5038304

Patiño, R., \& Sepúlveda, Y. (2017). Contexto empresarial colombiano y su relación con el gobierno corporativo, estudio de caso. Criterio Libre, 15(27). Recuperado de https://bbibliograficas.ucc.edu.co:2149/docview/2125 262178/fulltextPDF/32113DF596DF4937PQ/ ?accountid=44394 
Perdomo, J., Villa, L., \& Dueñas, S. (2017). Responsabilidad social corporativa y prácticas de ProPeace en Colombia. Sistema de Información Científica Redalyc, 33(58). Recuperado de https://www.redalyc.org/jatsRepo/2250/2 25056947004/index.html

Polanco, J., Ramírez F. \& Orozco M. (2016). Incidencia de estándares internacionales en la sostenibilidad corporativa: Una perspectiva de la alta dirección/International standards effect on corporate sustainability: A senior managers perspective. Estudios Gerenciales, 32(139), 181-192. Recuperado de https://bbibliograficas.ucc.edu.co:2149/d ocview $/ 1822463831$ ? accountid $=44394$

Puerta, F., Vergara, J., \& Huertas, N. (2018). Análisis financiero: enfoques en su evolución. Criterio Libre, 16(28). Recuperado de https://bbibliograficas.ucc.edu.co:2149/docview/2125263709/fulltextPDF/64C932328B3C4 $04 \mathrm{DPQ} / 1$ ? accountid $=44394$

Quintero, L. (2017). Responsabilidad social empresarial en las empresas del sector minorista. Clio America, 11(22). Recuperado de https://bbibliograficas.ucc.edu.co:2149/docview/2196580891/fulltextPDF/89EF53FAB67F4 $4 \mathrm{FDPQ} / 1$ ? accountid $=44394$

Ramírez, J. (2017). El sentido ético en la responsabilidad social: Economía, innovación y medio ambiente. Ensayos De Economía, 27(50), 15-36. Recuperado de https://bbibliograficas.ucc.edu.co:2149/docview/1925441196?a ccountid $=44394$

Rendón, B. (2018). Efecto convergencia en Colombia de las NCIF, en los establecimientos bancarios emisores de valores acciones. Entramado, 14(1). Recuperado de https://dialnet.unirioja.es/servlet/articulo?codigo=67421 05

Rodrigo, P., \& Arenas, D. (2014). La nueva gobernanza política y las colaboraciones intersectoriales para el desarrollo sostenible. Innovar, 24(53), 197-210. Retrieved from https://bbibliograficas.ucc.edu.co:2149/docview/16770 08810 ?accountid $=44394$

Rodríguez, L., Frías, J., \& García, R. (2014). El consejo de administración y las memorias de sostenibilidad. Revista de contabilidad, 17(1). Recuperado de https://dialnet.unirioja.es/servlet/articulo?codigo $=4692319$

Ruiz, M. (2018). Cualificación en normas internacionales de información financiera al iniciar la convergencia. Aglala, 9(1). Recuperado de https://dialnet.unirioja.es/servlet/articulo?codigo $=6832758$

Salamanca, C. (2016). Sustentabilidad y corporaciones autónomas regionales ambientales en Boyacá - Colombia. Apuntes del CENES, 35(61). Recuperado de https://bbibliograficas.ucc.edu.co:2149/docview/1768609054/f ulltextPDF/434F484C0B184BD0PQ/ 1 ?accountid $=44394$

Sanchis P. \& Rodríguez S. (2018). Responsabilidad social empresarial en banca. su aplicación al caso de la banca cooperativa. REVESCO: Revista De Estudios Cooperativos, 127, 24-227. Recuperado de: https://bbibliografic as.ucc.edu.co:2149/docview/2050585674?accountid=44394\&rfr_id=info\%3Axri\%2Fsid\%3Aprimo

Sepúlveda, Y., Valero, G., \& Bonilla, M. (2018). Un análisis de los estudios acerca de las memorias de sostenibilidad en Latinoamérica. Contabilidad y Negocios: Revista del Departamento Académico de Ciencias Administrativas. Recuperado de https://dialnet.unirioja.es/servlet/articulo?codigo $=6772588$

Solano, E. (2017). Análisis de los potenciales efectos en el reconocimiento de los activos de las pequeñas empresas de Cúcuta- Colombia por la implementación de la NIIF para Pymes. Respuestas, 22(2). Recuperado de https://d ialnet.unirioja.es/servlet/articulo?codigo $=6205730$

Solano, S., Plaza, P., \& Flores, S. (2015). Análisis de la divulgación de la información sobre la responsabilidad social corporativa en las empresas de transporte público urbano en España. Revista de contabilidad, 19(2). Recuperado de http://www.elsevier.es/es-revista-revista-contabilidad-spanish-accounting-review-368-pdf-S113848911500 0242

Toca, C. (2017). Aportes a la responsabilidad social. Revista Mexicana De Ciencias Políticas y Sociales, 62(230), 393-407. Recuperado de https://bbibliograficas.ucc.edu.co:2149/docview/1933861874?accountid=44394

Vaz, N., Ruiz, S., \& Fernández, B. (2018). El mercado de verificación de las memorias de sostenibilidad en España: un análisis desde la perspectiva de la demanda. Revista de Contabilidad, 21(1). Recuperado de https://www.scien cedirect.com/science/article/pii/S1138489117300195 
Vergara, R., Londoño, C., Pérez, N., \& Torres, R. (2015). La Adopción De Las Normas Internacionales De Información Financiera En Colombia. Panorama económico. Recuperado de https://revistas.unicartagena.edu .co/index.php/panoramaeconomico/article/view/1382

Villanueva, J., Zorio, A., \& García, M. (2015). Supervisores bursátiles, gobierno corporativo y cumplimiento de las NIIF: el caso de la CNMV. Innovar, 25(55). Recuperado de https://bbibliograficas.ucc.edu.co:2149/docview/ 1676943828/fulltextPDF/A43073E1C1B946E2PQ/1 ?accountid=44394

Volpentesta, J. (2017). Creación de un método de integración de grupos de interés al gobierno de las empresas Creación de un método de integración de partes interesadas para el gobierno corporativo. Estudios Gerenciales, 33(143). Recuperado de https://www.sciencedirect.com/science/article/pii/S0123592317300311

Witkowska, J. (2016). Responsabilidad social empresarial: Seleccionados aspectos teóricos y empíricos. Investigación económica y comparativa, 19(1). Recuperado de https://bbibliograficas.ucc.edu.co:2153/record/display.uri?eid=2-s2.0-84968867596\&origin=resultslist\&s ort $=$ plf-f\&src $=s \& s t 1=$ social + responsability $\& s t 2=\& s i d=5 \mathrm{ce} 8 \mathrm{cb} 0 \mathrm{a} 0 \mathrm{~b} 158 \mathrm{ce} 17 \mathrm{~b} 7054 \mathrm{a} 050 \mathrm{~d} 5382 \mathrm{e} \& \mathrm{sot}=\mathrm{b} \& \mathrm{sdt}$ $=\mathrm{b} \& \mathrm{sl}=36 \& \mathrm{~s}=\mathrm{TITLE}-\mathrm{ABS}-\mathrm{KEY} \% 28$ social+responsability\%29\&relpos=12\&citeCnt=3\&searchTerm $=$

La Universidad de Cuenca en Ecuador, conserva los derechos patrimoniales (copyright) de las obras publicadas, y favorece y permite la reutilización de las mismas bajo la licencia Creative Commons AtribuciónNoComercial-CompartirIgual 4.0 Internacional (CC BY-NC-SA 4.0), por lo cual se pueden copiar, usar, difundir, transmitir y exponer públicamente, siempre que: a. Se cite la autoría y fuente original de su publicación (revista, editorial, URL y DOI de la obra). b. No se usen para fines comerciales u onerosos. c. Se mencione la existencia y especificaciones de esta licencia de uso.

CC BY-NC-SA 